\title{
Applications of Soft Sets in BE-Algebras
}

\author{
Young Bae Jun ${ }^{1}$ and Sun Shin Ahn' \\ ${ }^{1}$ Department of Mathematics Education, Gyeongsang National University, Chinju 660-701, Republic of Korea \\ ${ }^{2}$ Department of Mathematics Education, Dongguk University, Seoul 100-715, Republic of Korea
}

Correspondence should be addressed to Sun Shin Ahn; sunshine@dongguk.edu

Received 11 December 2012; Accepted 14 March 2013

Academic Editor: Antonio M. Cegarra

Copyright (C) 2013 Y. B. Jun and S. S. Ahn. This is an open access article distributed under the Creative Commons Attribution License, which permits unrestricted use, distribution, and reproduction in any medium, provided the original work is properly cited.

The notion of intersectional soft subalgebras of a BE-algebra is introduced, and related properties are investigated. Characterization of an intersectional soft subalgebra is discussed. The problem of classifying intersectional soft subalgebras by their inclusive subalgebras will be solved.

\section{Introduction}

In 1966, Imai and Iséki [1] and Iséki [2] introduced two classes of abstract algebras: BCK-algebras and BCI-algebras. It is known that the class of BCK-algebras is a proper subclass of the class of BCI-algebras. Ma et al. studied $(\epsilon, \epsilon \vee q)$-tyle (interval-valued) fuzzy ideals in BCI-algebras and soft $R_{0}$ algebras (see [3-5]). As a generalization of a BCK-algebra, $\mathrm{H}$. S. Kim and Y. H. Kim [6] introduced the notion of a $B E-$ algebra and investigated several properties. In [7], Ahn and So introduced the notion of ideals in $B E$-algebras. They gave several descriptions of ideals in $B E$-algebras. Song et al. [8] considered the fuzzification of ideals in $B E$-algebras. They introduced the notion of fuzzy ideals in $B E$-algebras and investigated related properties. They gave characterizations of a fuzzy ideal in $B E$-algebras.

Various problems in system identification involve characteristics which are essentially nonprobabilistic in nature [9]. In response to this situation, Zadeh [10] introduced fuzzy set theory as an alternative to probability theory. Uncertainty is an attribute of information. In order to suggest a more general framework, the approach to uncertainty is outlined by Zadeh [11]. To solve complicated problem in economics, engineering, and environment, we cannot successfully use classical methods because of various uncertainties typical for those problems. There are three theories: theory of probability, theory of fuzzy sets, and the interval mathematics which we can consider as mathematical tools for dealing with uncertainties. But all these theories have their own difficulties. Uncertainties cannot be handled using traditional mathematical tools but may be dealt with using a wide range of existing theories such as probability theory, theory of (intuitionistic) fuzzy sets, theory of vague sets, theory of interval mathematics, and theory of rough sets. However, all of these theories have their own difficulties which are pointed out in [12]. Maji et al. [13] and Molodtsov [12] suggested that one reason for these difficulties may be due to the inadequacy of the parametrization tool of the theory. To overcome these difficulties, Molodtsov [12] introduced the concept of soft set as a new mathematical tool for dealing with uncertainties that is free from the difficulties that have troubled the usual theoretical approaches. Molodtsov pointed out several directions for the applications of soft sets. At present, works on the soft set theory are progressing rapidly. Maji et al. [13] described the application of soft set theory to a decision making problem. Maji et al. [14] also studied several operations on the theory of soft sets. Chen et al. [15] presented a new definition of soft set parametrization reduction and compared this definition to the related concept of attributes reduction in rough set theory. The algebraic structure of set theories dealing with uncertainties has been studied by some authors. Çağman et al. [16] introduced fuzzy parameterized (FP) soft sets and their related properties. They proposed a decision making method based on FP-soft set theory and provided an example which shows that the method can be successfully applied to the problems that 
contain uncertainties. Feng [17] considered the application of soft rough approximations in multicriteria group decision making problems. Aktaş and Çağman [18] studied the basic concepts of soft set theory and compared soft sets to fuzzy and rough sets, providing examples to clarify their differences. They also discussed the notion of soft groups. After that, many algebraic properties of soft sets are studied (see [1929]).

In this paper, we introduce the notion of int-soft subalgebras of a $B E$-algebra and investigate their properties. We consider characterization of an int-soft subalgebra, and solve the problem of classifying int-soft subalgebras by their inclusive subalgebras.

\section{Preliminaries}

Let $K(\tau)$ be the class of all algebras of type $\tau=(2,0)$. By a $B E$-algebra we mean a system $(X ; *, 1) \in K(\tau)$ in which the following axioms hold (see [6]):

$$
\begin{gathered}
(\forall x \in X)(x * x=1), \\
(\forall x \in X)(x * 1=1), \\
(\forall x \in X)(1 * x=x), \\
(\forall x, y, z \in X)(x *(y * z)=y *(x * z)) . \quad \text { (exchange) }
\end{gathered}
$$

A relation " $\leq$ " on a $B E$-algebra $X$ is defined by

$$
(\forall x, y \in X)(x \leq y \Longleftrightarrow x * y=1)
$$

A $B E$-algebra $(X ; *, 1)$ is said to be transitive (see [7]) if it satisfies

$$
(\forall x, y, z \in X)(y * z \leq(x * y) *(x * z)) .
$$

A $B E$-algebra $(X ; *, 1)$ is said to be self distributive (see [6]) if it satisfies

$$
(\forall x, y, z \in X)(x *(y * z)=(x * y) *(x * z)) .
$$

Note that every self distributive $B E$-algebra is transitive, but the converse is not true in general (see [7]).

A mapping $\mu: X \rightarrow Y$ of $B E$-algebras is called a homomorphism if $\mu(x * y)=\mu(x) * \mu(y)$ for all $x, y \in X$.

A soft set theory is introduced by Molodtsov [12], and Çağman and Enginoğlu [30] provided new definitions and various results on soft set theory.

In what follows, let $U$ be an initial universe set, and let $E$ be a set of parameters. Let $\mathscr{P}(U)$ denote the power set of $U$ and $A, B, C, \ldots \subseteq E$.

Definition 1 (see $[12,13])$. A soft set $(f, A)$ over $U$ is defined to be the set of ordered pairs

$$
(f, A):=\{(x, f(x)): x \in E, f(x) \in \mathscr{P}(U)\},
$$

where $f: E \rightarrow \mathscr{P}(U)$ such that $f(x)=\emptyset$ if $x \notin A$.
The function $f$ is called an approximate function of the soft set $(f, A)$.

In what follows, denote by $S(U)$ the set of all soft sets over $U$ by Çăgman and Enginoğlu [30].

For any soft sets $(f, X)$ and $(g, X)$ over $U$, we call $(f, X)$ a soft subset of $(g, X)$, denoted by $(f, X) \widetilde{\simeq}(g, X)$ if $f(x) \subseteq$ $g(x)$ for all $x \in X$. The soft $\left\{\begin{array}{c}\text { union } \\ \text { intersection }\end{array}\right\}$ of $(f, X)$ and $(g, X)$ is defined to be a soft set $\left\{\begin{array}{l}(f, X) \widetilde{\sim}(g, X)=(f \tilde{\cup} g, X) \\ (f, X) \tilde{\cap}(g, X)=(f \tilde{\cap} g, X)\end{array}\right\}$, where $\left\{\begin{array}{l}(f \widetilde{\cup} g)(x)=f(x) \cup g(x) \\ (f \widetilde{n} g)(x)=f(x) \cap g(x)\end{array}\right\}$ for all $x \in X$.

Definition 2 (see [31, 32]). Assume that $E$ has a binary operation $\hookrightarrow$. For any nonempty subset $A$ of $E$, a soft set $(f, A)$ over $U$ is said to be intersectional over $U$ if it satisfies

$$
(\forall x, y \in A)(x \hookrightarrow y \in A \Longrightarrow f(x) \cap f(y) \subseteq f(x \hookrightarrow y))
$$

For a soft set $(f, A)$ over $U$ and a subset $\gamma$ of $U$, the $\gamma$ inclusive set of $(f, A)$, denoted by $i_{A}(f ; \gamma)$, is defined to be the set

$$
i_{A}(f ; \gamma):=\{x \in A \mid \gamma \subseteq f(x)\}
$$

\section{Intersectional Soft Subalgebras}

In what follows, we take $E=X$ as a set of parameters, which is a $B E$-algebra under the operation “*” unless otherwise specified.

Definition 3. A soft set $(f, X)$ over $U$ is called an intersectional soft subalgebra (briefly, int-soft subalgebra) over $U$ if it satisfies

$$
(\forall x, y \in X)(f(x * y) \supseteq f(x) \cap f(y)) .
$$

Example 4. Let $E=X$ be the set of parameters where $X=$ $\{1, a, b, c, d\}$ is a $B E$-algebra with the following Cayley table:

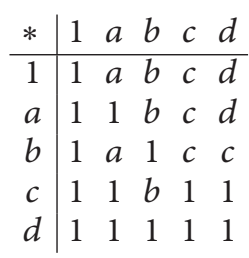

Let $(f, X)$ be a soft set over $U$ defined as follows:

$$
f: X \longrightarrow \mathscr{P}(U), \quad x \longmapsto \begin{cases}\gamma_{3} & \text { if } x=1, \\ \gamma_{1} & \text { if } x \in\{a, c, d\} \\ \gamma_{2} & \text { if } x=b\end{cases}
$$

where $\gamma_{1}, \gamma_{2}$, and $\gamma_{3}$ are subsets of $U$ with $\gamma_{1} \subsetneq \gamma_{2} \subsetneq \gamma_{3}$. It is easy to check that $(f, X)$ is an int-soft subalgebra over $U$. 
Example 5. Let $E=X$ be the set of parameters, and let $U=X$ be the initial universe set, where $X=\{1, a, b, c, d, 0\}$ is a $B E$ algebra [7] with the following Cayley table:

\begin{tabular}{l|llllll}
$*$ & 1 & $a$ & $b$ & $c$ & $d$ & 0 \\
\hline 1 & 1 & $a$ & $b$ & $c$ & $d$ & 0 \\
$a$ & 1 & 1 & $a$ & $c$ & $c$ & $d$ \\
$b$ & 1 & 1 & 1 & $c$ & $c$ & $c$ \\
$c$ & 1 & $a$ & $b$ & 1 & $a$ & $b$ \\
$d$ & 1 & 1 & $a$ & 1 & 1 & $a$ \\
0 & 1 & 1 & 1 & 1 & 1 & 1
\end{tabular}

Let $\left\{\gamma_{n} \mid n=1,2,3,4\right\}$ be a class of subsets of $U$ which is a poset under the following Hasse diagram:

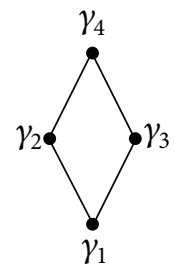

Let $(f, X)$ be a soft set over $U$ defined as follows:

$$
f: X \longrightarrow \mathscr{P}(U), \quad x \longmapsto \begin{cases}\gamma_{4} & \text { if } x=1, \\ \gamma_{1} & \text { if } x \in\{a, c, d\}, \\ \gamma_{2} & \text { if } x=b, \\ \gamma_{3} & \text { if } x=0 .\end{cases}
$$

It is easy to check that $(f, X)$ is an int-soft subalgebra over $U$.

Theorem 6. A soft set $(f, X)$ over $U$ is an int-soft subalgebra over $U$ if and only if $i_{X}(f ; \gamma)$ is a subalgebra of $X$ for all $\gamma \in$ $\mathscr{P}(U)$.

The subalgebra $i_{X}(f ; \gamma)$ in Theorem 6 is called the inclusive subalgebra of $X$.

Proof. Assume that $(f, X)$ is an int-soft subalgebra over $U$. Let $\gamma \in \mathscr{P}(U)$ and $x, y \in i_{X}(f ; \gamma)$. Then, $\gamma \subseteq f(x)$ and $\gamma \subseteq f(y)$. It follows from (11) that

$$
f(x * y) \supseteq f(x) \cap f(y) \supseteq \gamma,
$$

that is, $x * y \in i_{X}(f ; \gamma)$. Thus, $i_{X}(f ; \gamma)$ is a subalgebra of $X$.

Conversely, suppose that $i_{X}(f ; \gamma)$ is a subalgebra of $X$ for all $\gamma \in \mathscr{P}(U)$. Let $x, y \in X$ be such that $f(x)=\gamma_{x}$ and $f(y)=$ $\gamma_{y}$. Take $\gamma=\gamma_{x} \cap \gamma_{y}$. Then, $x, y \in i_{X}(f ; \gamma)$, and so $x * y \in$ $i_{X}(f ; \gamma)$ by assumption. Hence,

$$
f(x * y) \supseteq \gamma=\gamma_{x} \cap \gamma_{y}=f(x) \cap f(y) .
$$

Therefore, $(f, X)$ is an int-soft subalgebra over $U$.

Lemma 7. Every int-soft subalgebra $(f, X)$ over $U$ satisfies the following inclusion:

$$
(\forall x \in X)(f(x) \subseteq f(1))
$$

Proof. Using (1) and (11), we have

$$
f(1)=f(x * x) \supseteq f(x) \cap f(x)=f(x)
$$

for all $x \in X$.

Proposition 8. For any int-soft subalgebra $(f, X)$ over $U$, if a fixed element $x \in X$ satisfies $f(x)=f(1)$, then

$$
(\forall y \in X)(f(y) \subseteq f(x * y)) .
$$

Proof. Assume that a fixed element $x \in X$ satisfies $f(x)=$ $f(1)$. Then,

$$
f(y)=f(1) \cap f(y)=f(x) \cap f(y) \subseteq f(x * y)
$$

for all $y \in X$.

Proposition 9. Let $(f, X)$ be an int-soft subalgebra over $U$. If a fixed element $x \in X$ satisfies the following condition:

$$
(\forall y \in X)(f(y) \subseteq f(y * x)),
$$

then $f(x)=f(1)$.

Proof. Taking $y=1$ in (22) implies that $f(1) \subseteq f(1 * x)=$ $f(x)$ by (3). It follows from Lemma 7 that $f(x)=f(1)$.

For any $B E$-algebras $X$ and $Y$, let $\mu: X \rightarrow Y$ be a function and $(f, X)$, and let $(g, Y)$ be soft sets over $U$.

(1) The soft set

$\mu^{-1}(g, Y)=\left\{\left(x, \mu^{-1}(g)(x)\right): x \in X, \mu^{-1}(g)(x) \in \mathscr{P}(U)\right\}$,

where $\mu^{-1}(g)(x)=g(\mu(x))$, is called the soft preimage of $(g, Y)$ under $\mu$.

(2) The soft set

$$
\mu(f, X)=\{(y, \mu(f)(y)): y \in Y, \mu(f)(y) \in \mathscr{P}(U)\},
$$

where

$$
\mu(f)(y)= \begin{cases}\bigcup_{x \in \mu^{-1}(y)} f(x) & \text { if } \mu^{-1}(y) \neq \emptyset \\ \emptyset & \text { otherwise }\end{cases}
$$

is called the soft image of $(f, X)$ under $\mu$.

Proposition 10. For any BE-algebras $X$ and $Y$, let $\mu: X \rightarrow Y$ be a function. Then,

$$
(\forall(f, X) \in S(U))\left((f, X) \widetilde{\simeq} \mu^{-1}(\mu(f, X))\right) .
$$

Proof. Note that $\mu^{-1}(\mu(x)) \neq \emptyset$ for all $x \in X$. Hence,

$$
f(x) \subseteq \bigcup_{a \in \mu^{-1}(\mu(x))} f(a)=\mu(f)(\mu(x))=\mu^{-1}(\mu(f))(x)
$$

for all $x \in X$, and therefore (26) is valid. 
Theorem 11. Let $\mu: X \rightarrow Y$ be a homomorphism of BEalgebras and $(g, Y) \in S(U)$. If $(g, Y)$ is an int-soft subalgebra over $U$, then the soft preimage $\mu^{-1}(g, Y)$ of $(g, Y)$ under $\mu$ is also an int-soft subalgebra over $U$.

Proof. For any $x_{1}, x_{2} \in X$, we have

$$
\begin{aligned}
\mu^{-1}(g)\left(x_{1}\right) \cap \mu^{-1}(g)\left(x_{2}\right) & =g\left(\mu\left(x_{1}\right)\right) \cap g\left(\mu\left(x_{2}\right)\right) \\
& \subseteq g\left(\mu\left(x_{1}\right) * \mu\left(x_{2}\right)\right) \\
& =g\left(\mu\left(x_{1} * x_{2}\right)\right) \\
& =\mu^{-1}(g)\left(x_{1} * x_{2}\right) .
\end{aligned}
$$

Hence, $\mu^{-1}(g, Y)$ is also an int-soft subalgebra over $U$.

Theorem 12. Let $\mu: X \rightarrow Y$ be a homomorphism of BEalgebras and $(f, X) \in S(U)$. If $(f, X)$ is an int-soft subalgebra over $U$ and $\mu$ is injective, then the soft image $\mu(f, X)$ of $(f, X)$ under $\mu$ is also an int-soft subalgebra over $U$.

Proof. Let $y_{1}, y_{2} \in Y$. If at least one of $\mu^{-1}\left(y_{1}\right)$ and $\mu^{-1}\left(y_{2}\right)$ is empty, then the inclusion

$$
\mu(f)\left(y_{1}\right) \cap \mu(f)\left(y_{2}\right) \subseteq \mu(f)\left(y_{1} * y_{2}\right)
$$

is clear. Assume that $\mu^{-1}\left(y_{1}\right) \neq \emptyset$ and $\mu^{-1}\left(y_{2}\right) \neq \emptyset$. Since $\mu$ is injective, we have

$$
\begin{aligned}
\mu(f) & \left(y_{1}\right) \cap \mu(f)\left(y_{2}\right) \\
= & \left(\bigcup_{x_{1} \in \mu^{-1}\left(y_{1}\right)} f\left(x_{1}\right)\right) \cap\left(\bigcup_{x_{2} \in \mu^{-1}\left(y_{2}\right)} f\left(x_{2}\right)\right) \\
= & \bigcup_{\substack{x_{1} \in \mu^{-1}\left(y_{1}\right) \\
x_{2} \in \mu^{-1}\left(y_{2}\right)}}\left(f\left(x_{1}\right) \cap f\left(x_{2}\right)\right) \\
& \subseteq \bigcup_{\substack{x_{1} \in \mu^{-1}\left(y_{1}\right) \\
x_{2} \in \mu^{-1}\left(y_{2}\right)}}\left(f\left(x_{1} * x_{2}\right)\right) \\
= & \bigcup_{\substack{x \in \mu^{-1}\left(y_{1} * y_{2}\right) \\
=}} f(x) \\
= & \mu(f)\left(y_{1} * y_{2}\right) .
\end{aligned}
$$

Therefore, $\mu(f, X)$ is an int-soft subalgebra over $U$.

Theorem 13. Let $(f, X) \in S(U)$ and define a soft set $\left(f^{*}, X\right)$ over $U$ by

$$
f^{*}: X \longrightarrow \mathscr{P}(U), \quad x \longmapsto \begin{cases}f(x) & \text { if } x \in i_{X}(f ; \gamma), \\ \delta & \text { otherwise, }\end{cases}
$$

where $\gamma$ is any subset of $U$ and $\delta$ is a subset of $U$ satisfying $\delta \nsubseteq \bigcap_{x \notin i_{X}(f ; \gamma)} f(x)$. If $(f, X)$ is an int-soft subalgebra over $U$, then so is $\left(f^{*}, X\right)$.
Proof. If $(f, X)$ is an int-soft subalgebra over $U$, then $i_{X}(f ; \gamma)$ is a subalgebra of $X$ for all $\gamma \subseteq U$ by Theorem 6. Let $x, y \in X$. If $x, y \in i_{X}(f ; \gamma)$, then $x * y \in i_{X}(f ; \gamma)$. Hence,

$$
f^{*}(x * y)=f(x * y) \supseteq f(x) \cap f(y)=f^{*}(x) \cap f^{*}(y) .
$$

If $x \notin i_{X}(f ; \gamma)$ or $y \notin i_{X}(f ; \gamma)$, then $f^{*}(x)=\delta$ or $f^{*}(y)=\delta$. Thus,

$$
f^{*}(x * y) \supseteq \delta=f^{*}(x) \cap f^{*}(y) .
$$

Therefore, $\left(f^{*}, X\right)$ is an int-soft subalgebra over $U$.

Theorem 14. If $(f, X)$ and $(g, X)$ are int-soft subalgebras over $U$, then the soft intersection $(f, X) \widetilde{\cap}(g, X)$ of $(f, X)$ and $(g, X)$ is an int-soft subalgebra over $U$.

Proof. Let $x, y \in X$. Then,

$$
\begin{aligned}
(f \widetilde{\cap} g)(x * y) & =f(x * y) \cap g(x * y) \\
& \supseteq(f(x) \cap f(y)) \cap(g(x) \cap g(y)) \\
& =(f(x) \cap g(x)) \cap(f(y) \cap g(y)) \\
& =(f \widetilde{\cap} g)(x) \cap(f \widetilde{\cap} g)(y) .
\end{aligned}
$$

Hence, $(f, X) \widetilde{\cap}(g, X)$ is an int-soft subalgebra over $U$.

The following example shows that the soft union of intsoft subalgebras over $U$ may not be an int-soft subalgebra over $U$.

Example 15. Let $E=X$ be the set of parameters where $X=$ $\{1, a, b, c\}$ is a $B E$-algebra [7] with the following Cayley table:

$$
\begin{array}{l|llll}
* & 1 & a & b & c \\
\hline 1 & 1 & a & b & c \\
a & 1 & 1 & a & a \\
b & 1 & 1 & 1 & a \\
c & 1 & 1 & a & 1
\end{array}
$$

Let $(f, X)$ and $(g, X)$ be soft sets over $U$ defined, respectively, as follows:

$$
\begin{gathered}
f: X \longrightarrow \mathscr{P}(U), \quad x \longmapsto \begin{cases}\gamma_{5} & \text { if } x=1, \\
\gamma_{2} & \text { if } x=a, \\
\gamma_{1} & \text { if } x=b, \\
\gamma_{3} & \text { if } x=c,\end{cases} \\
g: X \longrightarrow \mathscr{P}(U), \quad x \longmapsto \begin{cases}\gamma_{4} & \text { if } x=1, \\
\gamma_{2} & \text { if } x=a, \\
\gamma_{3} & \text { if } x=b, \\
\gamma_{1} & \text { if } x=c,\end{cases}
\end{gathered}
$$

where $\gamma_{1}, \gamma_{2}, \gamma_{3}, \gamma_{4}$, and $\gamma_{5}$ are subsets of $U$ with $\gamma_{1} \mp \gamma_{2} \mp$ $\gamma_{3} \subsetneq \gamma_{4} \subsetneq \gamma_{5}$. It is easy to check that $(f, X)$ and $(g, X)$ are int-soft subalgebras over $U$. But $(f, X) \widetilde{U}(g, X)=(f \widetilde{\cup} g, X)$ is not an int-soft subalgebra over $U$, since $(f \widetilde{U} g)(c * b)=$ $(f \widetilde{\cup} g)(a)=\gamma_{2} \nsupseteq \gamma_{3}=(f \widetilde{\cup} g)(c) \cap(f \widetilde{\cup} g)(b)$. 
Theorem 16. Let $(f, X)$ be an int-soft subalgebra over $U$. Let $\gamma_{1}$ and $\gamma_{2}$ be subsets of $U$ such that $\gamma_{1} \mp \gamma_{2}$. If the $\gamma_{1}$-inclusive set of $(f, X)$ is equal to the $\gamma_{2}$-inclusive set of $(f, X)$, then there is no $x \in X$ such that $\gamma_{1} \subseteq f(x) \varsubsetneqq \gamma_{2}$.

Proof. Straightforward.

The converse of Theorem 16 is not true in general as seen in the following example.

Example 17. Let $E=X$ be the set of parameters, and let $U=X$ be the initial universe set where $X=\{1, a, b, c, d\}$ is a $B E$ algebra as in Example 4. Consider a soft set $(f, X)$ over $U$ which is given by

$$
f: X \longrightarrow \mathscr{P}(U), \quad x \longmapsto \begin{cases}X & \text { if } x=1, \\ \{1, a\} & \text { if } x \in\{a, c, d\}, \\ \{1, a, c\} & \text { if } x=b .\end{cases}
$$

Then, $(f, X)$ is an int-soft subalgebra over $U$. The $\gamma$-inclusive sets of $(f, X)$ are described as follows:

$$
i_{X}(f ; \gamma)= \begin{cases}X & \text { if } \gamma \in\{\emptyset,\{1\},\{a\},\{1, a\}\} \\ \{1, b\} & \text { if } \gamma \in\{\{c\},\{a, c\},\{1, a, c\}\}, \\ \{1\} & \text { if } \gamma \in \mathscr{P}(U) \backslash\{\emptyset,\{1\},\{a\},\{1, a\}, \\ & \quad\{c\},\{a, c\},\{1, a, c\}\} .\end{cases}
$$

If we take $\gamma_{1}=\{1, c\}$ and $\gamma_{2}=\{1, b, c\}$, then $\gamma_{1} \subsetneq \gamma_{2}$ and there is no $x \in X$ such that $\gamma_{1} \subseteq f(x) \mp \gamma_{2}$. But $i_{X}\left(f ; \gamma_{1}\right)=$ $\{1, b\} \neq\{1\}=i_{X}\left(f ; \gamma_{2}\right)$.

Theorem 18. Let $(f, X)$ be an int-soft subalgebra over $U$. Let $\gamma_{1}$ and $\gamma_{2}$ be subsets of $U$ such that $\gamma_{1} \mp \gamma_{2}$ and $\left\{\gamma_{1}, \gamma_{2}, f(x)\right\}$ are totally ordered by set inclusion for all $x \in X$. If there is no $x \in X$ such that $\gamma_{1} \subseteq f(x) \mp \gamma_{2}$, then the $\gamma_{1}$-inclusive set of $(f, X)$ is equal to the $\gamma_{2}$-inclusive set of $(f, X)$.

Proof. Since $\gamma_{1} \subsetneq \gamma_{2}$, we have $i_{X}\left(f ; \gamma_{2}\right) \subseteq i_{X}\left(f ; \gamma_{1}\right)$. If $x \in$ $i_{X}\left(f ; \gamma_{1}\right)$, then $\gamma_{1} \subseteq f(x)$. Since $\left\{\gamma_{1}, \gamma_{2}, f(x) \mid x \in X\right\}$ is totally ordered by inclusion and there is no $x \in X$ such that $\gamma_{1} \subseteq f(x) \subsetneq \gamma_{2}$, it follows that $\gamma_{2} \subseteq f(x)$, that is, $x \in i_{X}\left(f ; \gamma_{2}\right)$. Therefore, the $\gamma_{1}$-inclusive set of $(f, X)$ is equal to the $\gamma_{2}$ inclusive set of $(f, X)$.

Theorem 19. Let $(f, X)$ be a soft set over $U$ in which $\operatorname{Im}(f)$ is totally ordered by set inclusion. For each subset $\gamma$ of $\operatorname{Im}(f)$, if the $\gamma$-inclusive set of $(f, X)$ is a subalgebra of $X$, then $(f, X)$ is an int-soft subalgebra over $U$.

Proof. Let $x, y \in X$ be such that $f(x)=\gamma_{1}$ and $f(y)=\gamma_{2}$. Then, either $\gamma_{1} \subseteq \gamma_{2}$ or $\gamma_{2} \subseteq \gamma_{1}$. We may assume that $\gamma_{1} \subseteq \gamma_{2}$ without loss of generality. Then, $x \in i_{X}\left(f ; \gamma_{1}\right), y \in i_{X}\left(f ; \gamma_{2}\right)$, and $i_{X}\left(f ; \gamma_{2}\right) \subseteq i_{X}\left(f ; \gamma_{1}\right)$. Since $i_{X}\left(f ; \gamma_{1}\right)$ is a subalgebra of $X$, it follows that $x * y \in i_{X}\left(f ; \gamma_{1}\right)$ so that

$$
f(x * y) \supseteq \gamma_{1}=\gamma_{1} \cap \gamma_{2}=f(x) \cap f(y) .
$$

Therefore, $(f, X)$ is an int-soft subalgebra over $U$.
We have the following question.

Question. Let $(f, X)$ be an int-soft subalgebra over $U$. Does any subalgebra can be represented as a $\gamma$-inclusive set of $(f, X)$ ?

The following example shows that the answer to the question above is false.

Example 20. Let $E=X$ be the set of parameters, and let $U=$ $X$ be the initial universe set where $X=\{1, a, b, c\}$ is a $B E$ algebra as in Example 15. Consider a soft set $(f, X)$ over $U$ which is given by

$$
f: X \longrightarrow \mathscr{P}(U), \quad x \longmapsto \begin{cases}\{1, c\} & \text { if } x=1, \\ \{c\} & \text { if } x \in\{a, b, c\} .\end{cases}
$$

Then, $(f, X)$ is an int-soft subalgebra over $U$. The $\gamma$-inclusive sets of $(f, X)$ are described as follows:

$$
i_{X}(f ; \gamma)= \begin{cases}X & \text { if } \gamma \in\{\emptyset,\{c\}\}, \\ \{1\} & \text { if } \gamma \in\{\{1\},\{1, c\}\}, \\ \emptyset & \text { otherwise. }\end{cases}
$$

The subalgebra $\{1, b\}$ cannot be a $\gamma$-inclusive set $i_{X}(f ; \gamma)$, since there is no $\gamma \subseteq U$ such that $i_{X}(f ; \gamma)=\{1, b\}$.

However, we have the following theorem.

Theorem 21. Every subalgebra of a BE-algebra can be represented as a $\gamma$-inclusive set of an int-soft subalgebra.

Proof. Let $A$ be a subalgebra of a $B E$-algebra $X$. For a subset $\gamma$ of $U$, define a soft set $(f, X)$ over $U$ by

$$
f: X \longrightarrow \mathscr{P}(U), \quad x \longmapsto \begin{cases}\gamma & \text { if } x \in A, \\ \emptyset & \text { if } x \notin A .\end{cases}
$$

Obviously, $A=i_{X}(f ; \gamma)$. We now prove that $(f, X)$ is an intsoft subalgebra over $U$. Let $x, y \in X$. If $x, y \in A$, then $x * y \in$ $A$ because $A$ is a subalgebra of $X$. Hence, $f(x)=f(y)=$ $f(x * y)=\gamma$, and so $f(x) \cap f(y) \subseteq f(x * y)$. If $x \in A$ and $y \notin A$, then $f(x)=\gamma$ and $f(y)=\emptyset$ which imply that

$$
f(x) \cap f(y)=\gamma \cap \emptyset=\emptyset \subseteq f(x * y) .
$$

Similarly, if $x \notin A$ and $y \in A$, then $f(x) \cap f(y) \subseteq f(x * y)$. Obviously, if $x \notin A$ and $y \notin A$, then $f(x) \cap f(y) \subseteq f(x * y)$. Therefore, $(f, X)$ is an int-soft subalgebra over $U$.

Note that if $E=X$ is a finite $B E$-algebra, then the number of subalgebras of $X$ is finite whereas the number of $\gamma$-inclusive sets of an int-soft subalgebra over $U$ appears to be infinite. But, since every $\gamma$-inclusive set is indeed a subalgebra of $X$, not all these $\gamma$-inclusive sets are distinct. The next theorem characterizes this aspect.

Theorem 22. Let $(f, X)$ be an int-soft subalgebra over $U$ and let $\gamma_{1} \mp \gamma_{2} \subseteq U$ such that $\left\{\gamma_{1}, \gamma_{2}, f(x)\right\}$ is a chain for all $x \in X$. Two $\gamma$-inclusive sets $i_{X}\left(f ; \gamma_{1}\right)$ and $i_{X}\left(f ; \gamma_{2}\right)$ are equal if and only if there is no $x \in X$ such that $\gamma_{1} \mp f(x) \varsubsetneqq \gamma_{2}$. 
Proof. Let $\gamma_{1}$ and $\gamma_{2}$ be subsets of $U$ such that $i_{X}\left(f ; \gamma_{1}\right)=$ $i_{X}\left(f ; \gamma_{2}\right)$. Assume that there exists $x \in X$ such that $\gamma_{1} \varsubsetneqq$ $f(x) \varsubsetneqq \gamma_{2}$. Then, $i_{X}\left(f ; \gamma_{2}\right)$ is a proper subset of $i_{X}\left(f ; \gamma_{1}\right)$, which contradicts the hypothesis.

Conversely, suppose that there is no $x \in X$ such that $\gamma_{1} \subsetneq f(x) \subsetneq \gamma_{2}$. Obviously, $i_{X}\left(f ; \gamma_{2}\right) \subseteq i_{X}\left(f ; \gamma_{1}\right)$. If $x \in$ $i_{X}\left(f ; \gamma_{1}\right)$, then $\gamma_{1} \subseteq f(x)$. It follows from the assumption that $\gamma_{2} \subseteq f(x)$, that is, $x \in i_{X}\left(f ; \gamma_{2}\right)$. Therefore, $i_{X}\left(f ; \gamma_{1}\right)=$ $i_{X}\left(f ; \gamma_{2}\right)$.

Remark 23. As a consequence of Theorem 22, if $E=X$ is a finite $B E$-algebra, then the $\gamma$-inclusive sets of an int-soft subalgebra $(f, X)$ over $U$ form a chain. But $f(x) \subseteq f(1)$ for all $x \in X$. Therefore, $i_{X}\left(f ; \gamma_{0}\right)$, where $\gamma_{0}=f(1)$, is the smallest inclusive subalgebra but not always $i_{X}\left(f ; \gamma_{0}\right)=\{1\}$ as seen in the following example, and so we have the chain

$$
i_{X}\left(f ; \gamma_{0}\right) \subsetneq i_{X}\left(f ; \gamma_{1}\right) \subsetneq \cdots \subsetneq i_{X}\left(f ; \gamma_{r}\right)=X,
$$

where $\gamma_{0} \supsetneq \gamma_{1} \supsetneq \cdots \supsetneq \gamma_{r}$.

Example 24. Let $A$ be a subalgebra of a $B E$-algebra $X$ such that $A \neq\{1\}$. Let $(f ; X)$ be the int-soft subalgebra over $U$ which is given in the proof of Theorem 21. Then, $\operatorname{Im}(f)=$ $\{\emptyset, \gamma\}$. Further, the $\gamma$-inclusive sets of $(f, X)$ are $i_{X}(f ; \emptyset)=X$ and $i_{X}(f ; \gamma)=A$. Thus, we have $f(1)=\gamma$ but $i_{X}(f ; \gamma)=$ $A \neq\{1\}$.

Corollary 25. Let $E=X$ be a finite BE-algebra, and let $(f, X)$ be an int-soft subalgebra over $U$. If $\operatorname{Im}(f)=\left\{\gamma_{1}, \gamma_{2}, \ldots, \gamma_{n}\right\}$, then the family of $\gamma_{i}$-inclusive sets $i_{X}\left(f ; \gamma_{i}\right), 1 \leq i \leq n$, constitutes all the $\gamma$-inclusive sets of $(f, X)$.

Proof. Let $\gamma \subseteq U$ and $\gamma \notin \operatorname{Im}(f)$. If $\gamma_{i} \subsetneq \gamma \subsetneq \gamma_{j}$, where $\gamma_{i}, \gamma_{j} \in$ $\operatorname{Im}(f)$, then $i_{X}\left(f ; \gamma_{i}\right)=i_{X}\left(f ; \gamma_{j}\right)=i_{X}(f ; \gamma)$ by Theorem 22. If $\gamma \subsetneq \gamma_{r}$, where $\gamma_{r}$ is the least element (under the set inclusion) of $\operatorname{Im}(f)$, then $i_{X}\left(f ; \gamma_{r}\right)=X=i_{X}(f ; \gamma)$. Assume that $\gamma \supsetneq \gamma_{k}$, where $\gamma_{k}$ is the greatest element (under the set inclusion) of $\operatorname{Im}(f)$. If there is $x \in X$ such that $f(x)=\gamma_{x} \subseteq U$ and $\gamma_{k} \subsetneq$ $\gamma_{x} \subsetneq \gamma$, then $\gamma_{x} \in \operatorname{Im}(f)$. It is a contradiction. It follows from Theorem 22 that $i_{X}\left(f ; \gamma_{k}\right)=i_{X}(f ; \gamma)$. Thus, for any $\gamma \subseteq U$, the inclusive subalgebra is one of $\left\{i_{X}\left(f ; \gamma_{i}\right) \mid \gamma_{i} \in \operatorname{Im}(f)\right\}$.

The following example shows that two int-soft subalgebras over $U$ may have an identical family of $\gamma$-inclusive sets but the int-soft subalgebras over $U$ may not be equal.

Example 26. Let $E=X$ be the set of parameters, and let $U=$ $X$ be the initial universe set where $X=\{1, a, b, c\}$ is a $B E$ algebra as in Example 15. Consider a soft set $(f, X)$ over $U$ which is given by

$$
f: X \longrightarrow \mathscr{P}(U), \quad x \longmapsto \begin{cases}\gamma_{1} & \text { if } x=1, \\ \gamma_{2} & \text { if } x=a, \\ \gamma_{3} & \text { if } x \in\{b, c\}\end{cases}
$$

where $\gamma_{1} \supsetneq \gamma_{2} \supsetneq \gamma_{3}$ are subsets of $U$. It is easy to verify that $(f ; X)$ is an int-soft subalgebra over $U$. The $\gamma$-inclusive sets of $(f ; X)$ are $i_{X}\left(f ; \gamma_{1}\right)=\{1\}, i_{X}\left(f ; \gamma_{2}\right)=\{1, a\}$ and $i_{X}\left(f ; \gamma_{3}\right)=X$. Now let $\delta_{1}, \delta_{2}$, and $\delta_{3}$ be subsets of $U$ such that $\delta_{1} \supsetneq \delta_{2} \supsetneq \delta_{3}$ and $\delta_{i} \neq \gamma_{j}$ for $i=1,2,3$ and $j=1,2,3$. Define a soft set $(g ; X)$ over $U$ as follows:

$$
g: X \longrightarrow \mathscr{P}(U), \quad x \longmapsto \begin{cases}\delta_{1} & \text { if } x=1, \\ \delta_{2} & \text { if } x=a, \\ \delta_{3} & \text { if } x \in\{b, c\} .\end{cases}
$$

Then, $(g, X)$ is an int-soft subalgebra over $U$, and the $\gamma$ inclusive sets of $(g ; X)$ are $i_{X}\left(g ; \delta_{1}\right)=\{1\}, i_{X}\left(g ; \delta_{2}\right)=\{1, a\}$ and $i_{X}\left(g ; \delta_{3}\right)=X$. Hence, the two int-soft subalgebras $(f, X)$ and $(g, X)$ over $U$ have an identical family of $\gamma$-inclusive sets. However, it is clear that $(f, X)$ is not equal to $(g, X)$.

Lemma 27. Let $E=X$ be a finite BE-algebra, and let $(f, X)$ be an int-soft subalgebra over $U$. If $\gamma_{i}$ and $\gamma_{j}$ are elements of $\operatorname{Im}(f)$ such that $i_{X}\left(f ; \gamma_{i}\right)=i_{X}\left(f ; \gamma_{j}\right)$, then $\gamma_{i}=\gamma_{j}$.

Proof. Straightforward.

Theorem 28. Let $E=X$ be a finite BE-algebra, and let $(f, X)$ and $(g, X)$ be two int-soft subalgebras over $U$ having the identical family of $\gamma$-inclusive sets. If $\operatorname{Im}(f)=\left\{\gamma_{0}, \gamma_{1}, \ldots, \gamma_{r}\right\}$ and $\operatorname{Im}(g)=\left\{\delta_{0}, \delta_{1}, \ldots, \delta_{k}\right\}$, where

$$
\gamma_{0} \supsetneq \gamma_{1} \supsetneq \cdots \supsetneq \gamma_{r}, \quad \delta_{0} \supsetneq \delta_{1} \supsetneq \cdots \supsetneq \delta_{k},
$$

then we have
(1) $r=k$,
(2) $i_{X}\left(f ; \gamma_{i}\right)=i_{X}\left(g ; \delta_{i}\right), 0 \leq i \leq r$,
(3) $(\forall x \in X)\left(f(x)=\gamma_{i} \Rightarrow g(x)=\delta_{i}, 0 \leq i \leq r\right)$.

Proof. Corollary 25 implies that the only $\gamma$-inclusive sets of $(f, X)$ and $(g, X)$ are the two families $i_{X}\left(f ; \gamma_{i}\right)$ and $i_{X}\left(g ; \delta_{i}\right)$. Since $(f, X)$ and $(g, X)$ have the same family of $\gamma$-inclusive sets, we have $r=k$ which proves (1).

(2) Using (1) and Remark 23, we have two chains of $\gamma$ inclusive sets:

$$
\begin{aligned}
& i_{X}\left(f ; \gamma_{0}\right) \subsetneq i_{X}\left(f ; \gamma_{1}\right) \subsetneq \cdots \subsetneq i_{X}\left(f ; \gamma_{r}\right)=X, \\
& i_{X}\left(g ; \delta_{0}\right) \subsetneq i_{X}\left(g ; \delta_{1}\right) \subsetneq \cdots \subsetneq i_{X}\left(g ; \delta_{r}\right)=X .
\end{aligned}
$$

Clearly, we have

$$
\begin{aligned}
& \left(\forall \gamma_{i}, \gamma_{j} \in \operatorname{Im}(f)\right)\left(\gamma_{i} \supsetneq \gamma_{j} \Longrightarrow i_{X}\left(f ; \gamma_{i}\right) \varsubsetneqq i_{X}\left(f ; \gamma_{j}\right)\right), \\
& \left(\forall \delta_{i}, \delta_{j} \in \operatorname{Im}(g)\right)\left(\delta_{i} \supsetneq \delta_{j} \Longrightarrow i_{X}\left(g ; \delta_{i}\right) \varsubsetneqq i_{X}\left(g ; \delta_{j}\right)\right) .
\end{aligned}
$$

Since two families of $\gamma$-inclusive sets are identical, it is clear that $i_{X}\left(f ; \gamma_{0}\right)=i_{X}\left(g ; \delta_{0}\right)$. By hypothesis, $i_{X}\left(f ; \gamma_{1}\right)=i_{X}\left(g ; \delta_{j}\right)$ for some $j>0$. Assume that $i_{X}\left(f ; \gamma_{1}\right) \neq i_{X}\left(g ; \delta_{1}\right)$. Then, $i_{X}\left(f ; \gamma_{1}\right)=i_{X}\left(g ; \delta_{j}\right)$ for some $j>1$, and $i_{X}\left(g ; \delta_{1}\right)=i_{X}\left(f ; \gamma_{i}\right)$ for some $\gamma_{i} \subsetneq \gamma_{1}$. Thus, by (49) and (50), we have $i_{X}\left(g ; \delta_{j}\right)=$ $i_{X}\left(f ; \gamma_{1}\right) \subsetneq i_{X}\left(f ; \gamma_{i}\right)$ and $i_{X}\left(f ; \gamma_{i}\right)=i_{X}\left(g ; \delta_{1}\right) \subsetneq i_{X}\left(g ; \delta_{j}\right)$. This is a contradiction, and so $i_{X}\left(f ; \gamma_{1}\right)=i_{X}\left(g ; \delta_{1}\right)$. By mathematical induction on $i, 0 \leq i \leq r$, we finally obtain $i_{X}\left(f ; \gamma_{i}\right)=i_{X}\left(g ; \delta_{i}\right), 0 \leq i \leq r$.

(3) Let $x \in X$ be such that $f(x)=\gamma_{i}$ and $g(x)=\delta_{j}$, where $0 \leq i \leq r$ and $0 \leq j \leq r$. It is sufficient to show that 
$\delta_{j}=\delta_{i}$. Now, $x \in i_{X}\left(f ; \gamma_{i}\right)=i_{X}\left(g ; \delta_{i}\right)$ implies that $g(x)=$ $\delta_{j} \supsetneq \delta_{i}$. This gives from (50) that $i_{X}\left(g ; \delta_{j}\right) \subsetneq i_{X}\left(g ; \delta_{i}\right)$. Since $x \in i_{X}\left(g ; \delta_{j}\right)$, it follows from (2) that $x \in i_{X}\left(f ; \gamma_{j}\right)$ and so $f(x)=\gamma_{i} \supsetneq \gamma_{j}$. Hence, $i_{X}\left(f ; \gamma_{i}\right) \varsubsetneqq i_{X}\left(f ; \gamma_{j}\right)$ by (49). Using (2), we have $i_{X}\left(g ; \delta_{i}\right)=i_{X}\left(f ; \gamma_{i}\right) \varsubsetneqq i_{X}\left(f ; \gamma_{j}\right)=i_{X}\left(g ; \delta_{j}\right)$. Thus, $i_{X}\left(g ; \delta_{i}\right)=i_{X}\left(g ; \delta_{j}\right)$, and so $\delta_{j}=\delta_{i}$. This completes the proof.

Theorem 29. Let $E=X$ be a BE-algebra. Given any chain of subalgebras

$$
A_{0} \subsetneq A_{1} \subsetneq A_{2} \subsetneq \cdots \varsubsetneqq A_{r}=X,
$$

there exists an int-soft subalgebra over $U$ whose $\gamma$-inclusive sets are exactly the subalgebras of this chain.

Proof. Consider a class $\left\{\gamma_{i} \mid i=0,1,2, \ldots, r\right\}$ of subsets of $U$ such that

$$
\gamma_{0} \supsetneq \gamma_{1} \supsetneq \cdots \supsetneq \gamma_{r} .
$$

Define a soft set $(f, X): E \rightarrow \mathscr{P}(U)$ by $f\left(A_{0}\right)=\gamma_{0}$ and $f\left(A_{i}-A_{i-1}\right)=\gamma_{i}, 0<i \leq r$. We will prove that $(f, X)$ is an intersectional soft $B E$-algebra over $U$. Note that if $x \in A_{i}$, then $\gamma_{i} \subseteq f(x)$. If $x \in A_{i}$, then either $x \in A_{i}-A_{j}$ or $x \in A_{j}$ for $i>j$. Thus, if $x \in A_{i}-A_{j}$, then $f(x)=\gamma_{i}$. If $x \in A_{j}$, then $\gamma_{i} \subseteq \gamma_{j} \subseteq f(x)$. Let $x, y \in X$. We distinguish two cases as follows:

Case 1. Let $x, y \in A_{i}-A_{i-1}$. Then, $f(x)=\gamma_{i}=f(y)$. Since $A_{i}$ is a subalgebra, we have $x * y \in A_{i}$, and so either $x * y \in$ $A_{i}-A_{i-1}$ or $x * y \in A_{i-1}$. In any case we know that

$$
f(x) \cap f(y)=\gamma_{i} \subseteq f(x * y) .
$$

Case 2. For $i>j$, let $x \in A_{i}-A_{i-1}$ and $y \in A_{j}-A_{j-1}$. Then, $f(x)=\gamma_{i}, f(y)=\gamma_{j}$ and $x * y \in A_{i}$. It follows that

$$
f(x) \cap f(y)=\gamma_{i} \cap \gamma_{j}=\gamma_{i} \subseteq f(x * y) .
$$

Hence, we conclude that $(f, X)$ is an intersectional soft $B E$-algebra over $U$. From the definition of $(f, X)$, we have $\operatorname{Im}(f)=\left\{\gamma_{0}, \gamma_{1}, \ldots, \gamma_{r}\right\}$. Thus, the $\gamma$-inclusive sets of $X$ are given by the chain of subalgebras

$$
i_{X}\left(f ; \gamma_{0}\right) \varsubsetneqq i_{X}\left(f ; \gamma_{1}\right) \varsubsetneqq \cdots \varsubsetneqq i_{X}\left(f ; \gamma_{r}\right)=X .
$$

Now, $i_{X}\left(f ; \gamma_{0}\right)=\left\{x \in X \mid \gamma_{0} \subseteq f(x)\right\}=A_{0}$. Finally, we prove that $i_{X}\left(f ; \gamma_{i}\right)=A_{i}$ for $0<i \leq r$. Clearly $A_{i} \subseteq i_{X}\left(f ; \gamma_{i}\right)$. If $x \in i_{X}\left(f ; \gamma_{i}\right)$, then $\gamma_{i} \subseteq f(x)$, and so $x \notin A_{j}$ for $j>i$. Hence, $f(x) \in\left\{\gamma_{1}, \gamma_{2}, \ldots, \gamma_{r}\right\}$, and thus $x \in A_{k}$ for some $k \leq i$. Since $A_{k} \subseteq A_{i}$, we have $x \in A_{i}$, and so $i_{X}\left(f ; \gamma_{i}\right)=A_{i}$ for $0 \leq i \leq r$. This completes the proof.

Theorem 29 is illustrated as an example.

Example 30. Let $U=\mathbb{Z}$ be the initial universe set, and let $E=X$ be the set of parameters where $X=\{1, a, b, c, d, 0\}$ is a $B E$-algebra as in Example 5. Consider subalgebras $A_{1}=\{1\}$,
$A_{2}=\{1, a\}, A_{3}=\{1, a, c, d\}$, and $A_{4}=X$. Then, $A_{1} \subsetneq A_{2} \subsetneq$ $A_{3} \varsubsetneqq A_{4}$. Define a soft set $(f, X)$ over $U$ by

$$
f: X \longrightarrow \mathscr{P}(U), \quad x \longmapsto \begin{cases}\mathbb{Z} & \text { if } x=1, \\ 2 \mathbb{Z} & \text { if } x=a, \\ 4 \mathbb{Z} & \text { if } x \in\{c, d\}, \\ 8 \mathbb{Z} & \text { if } x \in\{b, 0\} .\end{cases}
$$

Then, $(f, X)$ is an int-soft subalgebra over $U$ with $i_{X}(f ; \mathbb{Z})=$ $\{1\}=A_{1}, i_{X}(f ; 2 \mathbb{Z})=\{1, a\}=A_{2}, i_{X}(f ; 4 \mathbb{Z})=\{1, a, c, d\}=$ $A_{3}$, and $i_{X}(f ; 8 \mathbb{Z})=X=A_{4}$.

Theorem 31. Let $(f, X)$ be a soft set over $U$, and let $\gamma$ be a subset of $U$. Define a soft set $\left(f^{*}, X\right)$ over $U$ by

$$
f^{*}: X \longrightarrow \mathscr{P}(U), \quad x \longmapsto \begin{cases}f(x) & \text { if } x \in i_{X}(f ; \gamma), \\ \emptyset & \text { otherwise. }\end{cases}
$$

If $(f, X)$ is an int-soft subalgebra over $U$, then so is $\left(f^{*}, X\right)$.

Proof. Let $x, y \in X$. If $x, y \in i_{X}(f ; \gamma)$, then $x * y \in i_{X}(f ; \gamma)$, and so

$$
f^{*}(x * y)=f(x * y) \supseteq f(x) \cap f(y)=f^{*}(x) \cap f^{*}(y) .
$$

If $x \notin i_{X}(f ; \gamma)$ or $y \notin i_{X}(f ; \gamma)$, then $f^{*}(x)=\emptyset$ or $f^{*}(y)=\emptyset$. Hence,

$$
f^{*}(x) \cap f^{*}(y)=\emptyset \subseteq f^{*}(x * y) .
$$

Therefore, $\left(f^{*}, X\right)$ is an int-soft subalgebra over $U$.

\section{Conclusion}

Using the notion of int-soft sets, we have introduced the concept of int-soft subalgebras in $B E$-algebras and investigated related properties. We have considered characterization of an int-soft subalgebra and solved the problem of classifying int-soft subalgebras by their inclusive subalgebras. We have shown that

(1) every soft image of an int-soft subalgebra is also an int-soft subalgebra;

(2) the soft intersection of int-soft subalgebras is an intsoft subalgebra.

We have made a new int-soft subalgebra from the old one. Work is ongoing. Some important issues for future work are as follows:

(1) to develop strategies for obtaining more valuable results,

(2) to apply these notions and results for studying related notions in other (soft) algebraic structures,

(3) to study the soft set application in ideal and filter theory of $B E$-algebras. 


\section{Acknowledgments}

The authors wish to thank the anonymous reviewer(s) for their valuable suggestions. This work (RPP-2012-021) was supported by the fund of Research Promotion Program, Gyeongsang National University, 2012.

\section{References}

[1] Y. Imai and K. Iséki, "On axiom systems of propositional calculi. XIV," Proceedings of the Japan Academy, vol. 42, pp. 19-22, 1966.

[2] K. Iséki, "An algebra related with a propositional calculus," Proceedings of the Japan Academy, vol. 42, pp. 26-29, 1966.

[3] X. Ma, J. Zhan, and Y. B. Jun, "New types of fuzzy ideals of BCIalgebras," Neural Computing and Applications, vol. 21, pp. S19S27, 2012.

[4] X. Ma, J. Zhan, and Y. B. Jun, "Soft $R_{0}$-algebras based on fuzzy sets," Journal of Multiple-Valued Logic and Soft Computing, vol. 19, pp. 547-563, 2012.

[5] X. Ma, J. Zhan, B. Davvaz, and Y. B. Jun, "Some kinds of $(\epsilon, \epsilon$ $\mathrm{Vq}$ )-interval-valued fuzzy ideals of BCI-algebras," Information Sciences, vol. 178, no. 19, pp. 3738-3754, 2008.

[6] H. S. Kim and Y. H. Kim, "On BE-algebras," Scientiae Mathematicae Japonicae, vol. 66, no. 1, pp. 113-116, 2007.

[7] S. S. Ahn and K. S. So, "On ideals and upper sets in BE-algebras," Scientiae Mathematicae Japonicae, vol. 68, no. 2, pp. 279-285, 2008.

[8] S. Z. Song, Y. B. Jun, and K. J. Lee, "Fuzzy ideals in BE-algebras," Bulletin of the Malaysian Mathematical Sciences Society, vol. 33, no. 1, pp. 147-153, 2010.

[9] L. A. Zadeh, "From circuit theory to system theory," Proceedings of the Institute of Radio Engineers, vol. 50, pp. 856-865, 1962.

[10] L. A. Zadeh, "Fuzzy sets," Information and Computation, vol. 8, pp. 338-353, 1965.

[11] L. A. Zadeh, "Toward a generalized theory of uncertainty (GTU) - an outline," Information Sciences, vol. 172, no. 1-2, pp. 1-40, 2005.

[12] D. Molodtsov, "Soft set theory-first results," Computers \& Mathematics with Applications, vol. 37, no. 4-5, pp. 19-31, 1999.

[13] P. K. Maji, A. R. Roy, and R. Biswas, "An application of soft sets in a decision making problem," Computers \& Mathematics with Applications, vol. 44, no. 8-9, pp. 1077-1083, 2002.

[14] P. K. Maji, R. Biswas, and A. R. Roy, "Soft set theory," Computers \& Mathematics with Applications, vol. 45, no. 4-5, pp. 555-562, 2003.

[15] D. Chen, E. C. C. Tsang, D. S. Yeung, and X. Wang, "The parameterization reduction of soft sets and its applications," Computers \& Mathematics with Applications, vol. 49, no. 5-6, pp. 757-763, 2005.

[16] N. Çağman, F. Çitak, and S. Enginoglu, "FP-soft set theory and its applications," Annals of Fuzzy Mathematics and Informatics, vol. 2, no. 2, pp. 219-226, 2011.

[17] F. Feng, "Soft rough sets applied to multicriteria group decision making," Annals of Fuzzy Mathematics and Informatics, vol. 2, no. 1, pp. 69-80, 2011.

[18] H. Aktaş and N. Çağman, "Soft sets and soft groups," Information Sciences, vol. 177, no. 13, pp. 2726-2735, 2007.

[19] U. Acar, F. Koyuncu, and B. Tanay, "Soft sets and soft rings," Computers \& Mathematics with Applications, vol. 59, no. 11, pp. 3458-3463, 2010.
[20] A. O. Atagün and A. Sezgin, "Soft substructures of rings, fields and modules," Computers \& Mathematics with Applications, vol. 61, no. 3, pp. 592-601, 2011.

[21] F. Feng, Y. B. Jun, and X. Zhao, "Soft semirings," Computers \& Mathematics with Applications, vol. 56, no. 10, pp. 2621-2628, 2008.

[22] Y. B. Jun, "Soft BCK/BCI-algebras," Computers \& Mathematics with Applications, vol. 56, no. 5, pp. 1408-1413, 2008.

[23] Y. B. Jun, H. S. Kim, and J. Neggers, "Pseudo $d$-algebras," Information Sciences, vol. 179, no. 11, pp. 1751-1759, 2009.

[24] Y. B. Jun, K. J. Lee, and C. H. Park, "Soft set theory applied to ideals in $d$-algebras," Computers \& Mathematics with Applications, vol. 57, no. 3, pp. 367-378, 2009.

[25] Y. B. Jun, K. J. Lee, and J. Zhan, "Soft p-ideals of soft BCIalgebras," Computers \& Mathematics with Applications, vol. 58, no. 10, pp. 2060-2068, 2009.

[26] Y. B. Jun, K. J. Lee, and A. Khan, "Soft ordered semigroups," Mathematical Logic Quarterly, vol. 56, no. 1, pp. 42-50, 2010.

[27] Y. B. Jun and C. H. Park, "Applications of soft sets in ideal theory of BCK/BCI-algebras," Information Sciences, vol. 178, no. 11, pp. 2466-2475, 2008.

[28] C. H. Park, Y. B. Jun, and M. A. Öztürk, "Soft WS-algebras," Communications of the Korean Mathematical Society, vol. 23, no. 3, pp. 313-324, 2008.

[29] J. Zhan and Y. B. Jun, "Soft BE-algebras based on fuzzy sets," Computers \& Mathematics with Applications, vol. 59, no. 6, pp. 2037-2046, 2010.

[30] N. Çağman and S. Enginoğlu, "Soft set theory and uni-int decision making," European Journal of Operational Research, vol. 207, no. 2, pp. 848-855, 2010.

[31] Y. B. Jun, M. S. Kang, and K. J. Lee, "Intersectional soft sets and applications to $\mathrm{BCK} / \mathrm{BCI}$-algebras," Communications of the Korean Mathematical Society, vol. 28, pp. 11-12, 2013.

[32] Y. B. Jun, K. J. Lee, and E. H. Roh, "Intersectional soft BCK/BCIideals," Annals of Fuzzy Mathematics and Informatics, vol. 4, no. 1, pp. 1-7, 2012. 


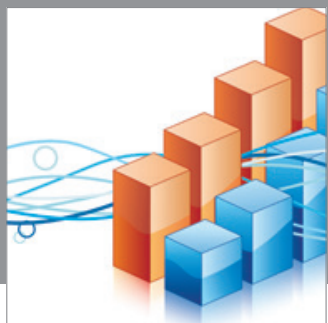

Advances in

Operations Research

mansans

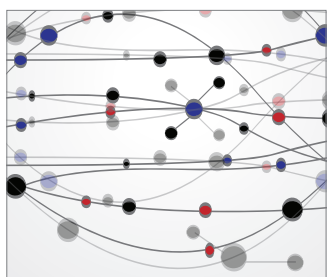

The Scientific World Journal
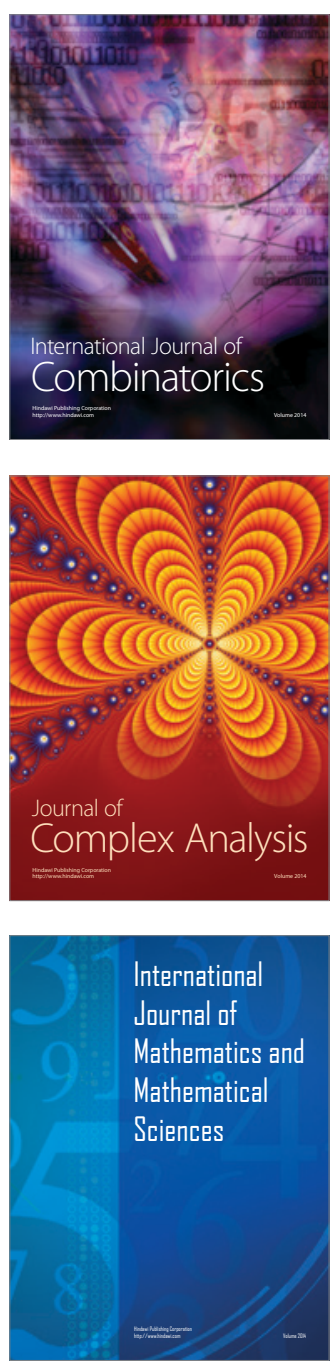
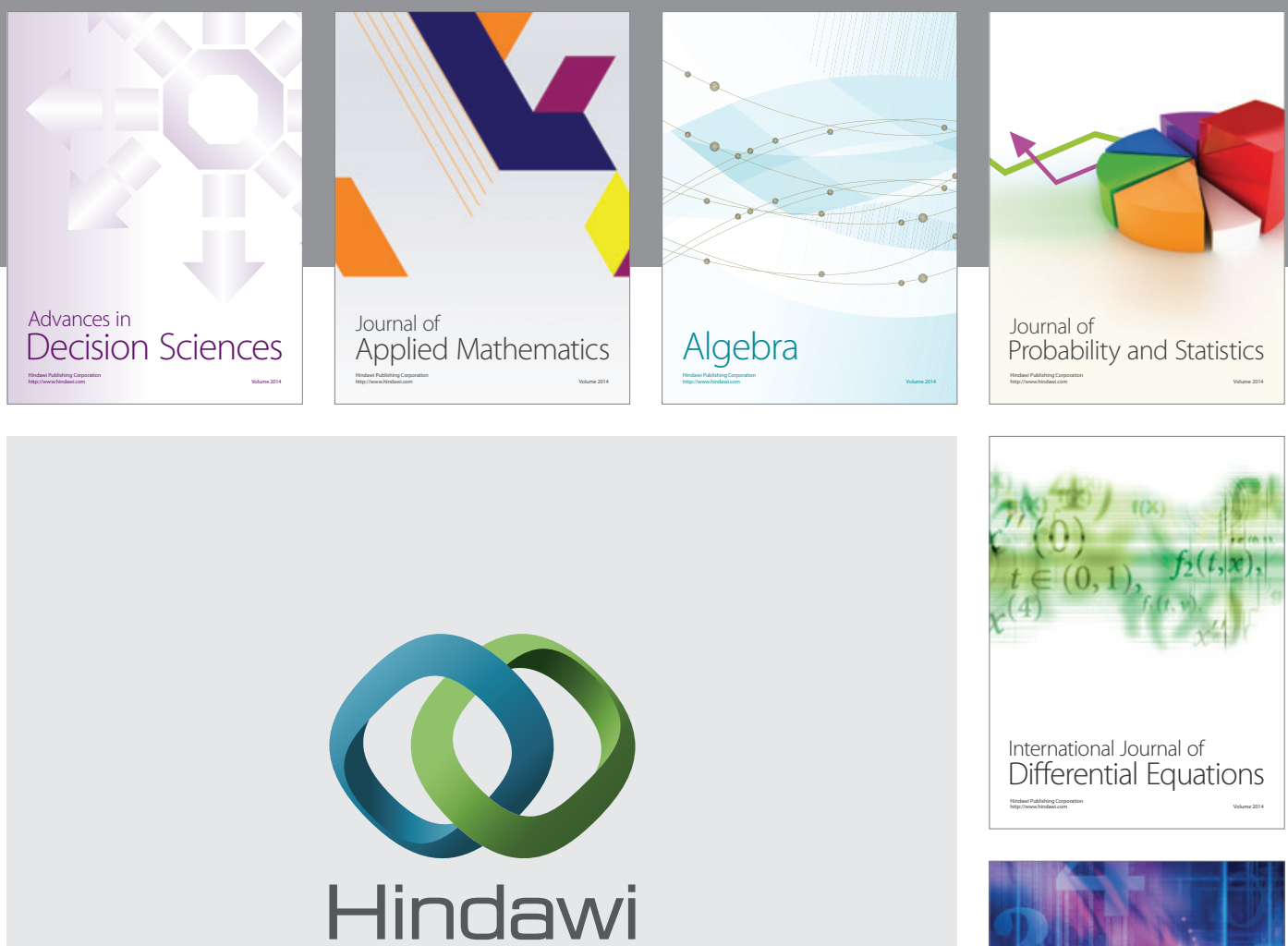

Submit your manuscripts at http://www.hindawi.com
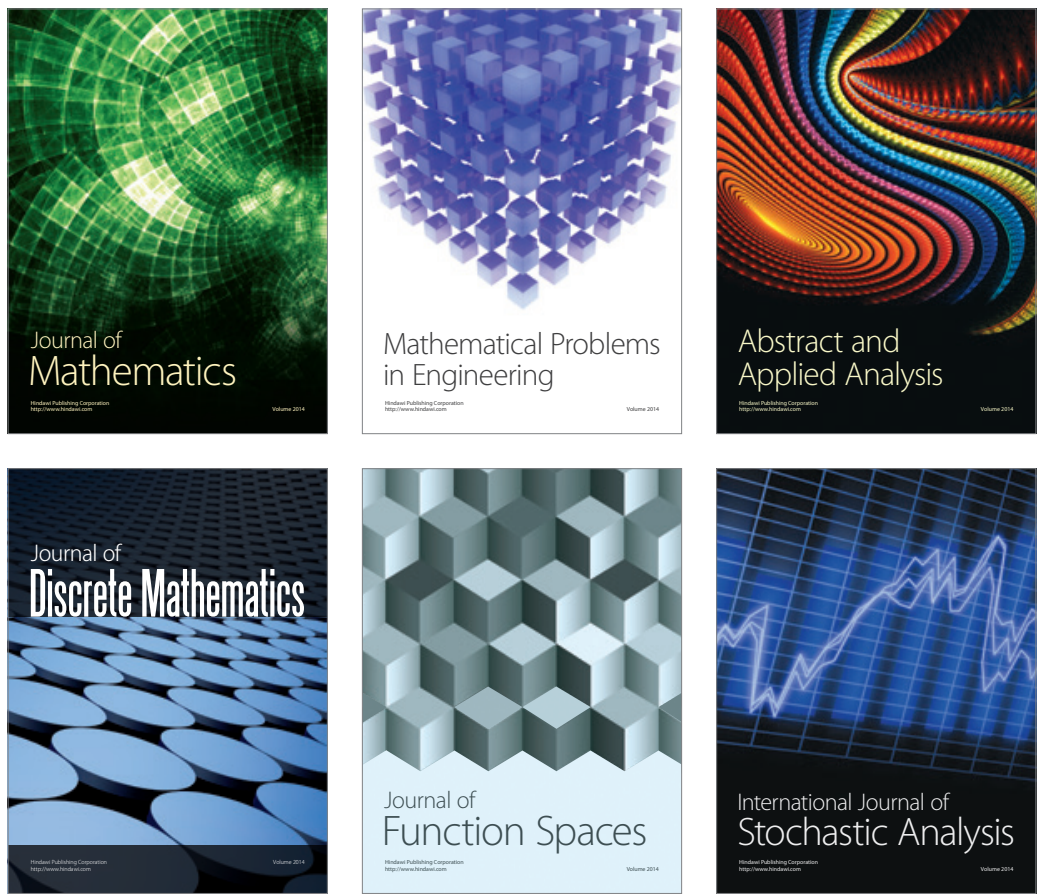

Journal of

Function Spaces

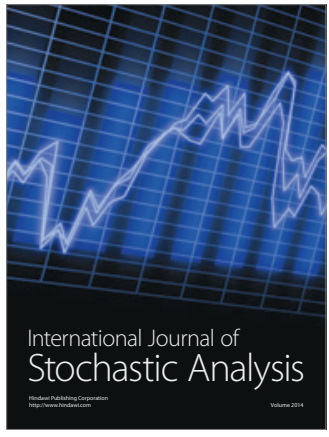

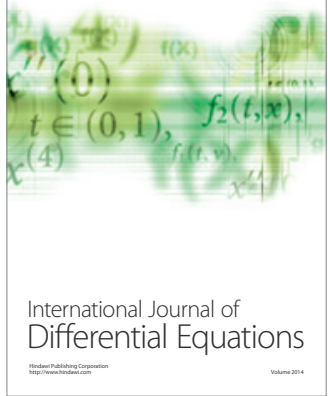
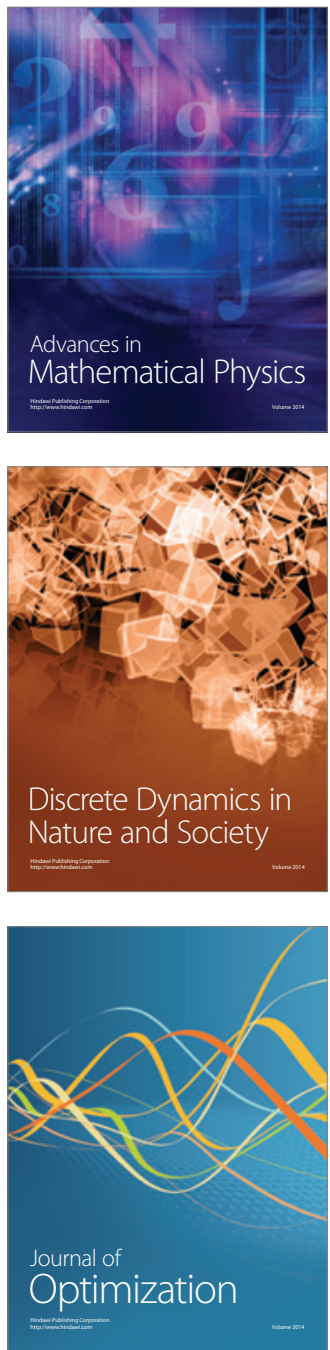